\title{
An Analysis of the Impact of Transaction Cost on the Borrower's Refinancing Decisions
}

\author{
Di Jin, Jin Zheng, Nan Zhang, and Siwei Gan
}

\begin{abstract}
Assuming that the market interest rate follows the Vasicek model, this paper focuses on finding an optimal refinancing interest rate boundary where a proportional transaction fee is charged when the mortgage borrower decides to leverage on the refinancing strategy to lower his financial cost. An optimal refinancing premium is introduced by risk-neutral analysis, then is used to recursively determine the minimization of discounted payment streams of the mortgage contract. Intriguing properties of the optimal refinancing boundary are discovered with numerical simulations.
\end{abstract}

Index Terms-Mortgage refinancing, refinancing value, transaction fee, risk-neutral pricing.

\section{INTRODUCTION}

Residential mortgage contract is one of the most-widely traded financial instruments in financial markets. Many existing work focuses on the value of residential mortgages with various analytical and numerical methods [1]-[6], and the default contingencies of mortgage-backed securities as in [7], for example. The importance of such studies to market participants as well as macro-economic policy makers has been well discussed and documented, where references in this regard can be found in [4], [8]-[9], for instance. While the topic of residential mortgages can be very general and broad, the goal of the current work is very specific: we aim to investigate how transaction cost will affect the optimal financial decision that the mortgage borrower can make when alternative borrowing rate is available in the market, provided that refinancing is allowed by the contract that the borrower currently holds. We admit that a direct approach to finding the borrower's optimal decisions with refinancing choices taken into account is not easy and literature of such is scarce although studies on mortgages allowing contingent claims are ample in general. On the other hand, a Monte Carlo simulation based numerical scheme is introduced and successfully implemented in [10] and [11] to address the problem, where several interesting characteristics pertaining to the borrower's optimal financial decisions are presented. In [10], the authors emphasized the difference between prepayment and refinancing and obtained a desirable refinancing time for mortgage borrowers to minimize the total payment by assuming that the market always provides

Manuscript received May 20, 2013; revised July 24, 2013.

Jin Di is with the Department of Mathematics, Xi'an Jiaotong University, China (email: jindijindy@stu.xjtu.edu.cn).

Jin Zheng is with the Department of Mathematical Sciences, University of Liverpool, U.K (email: jin.zheng@liverpool.ac.uk

Nan Zhang is with the Department of Computer Science and Software Engineering, Xi'an Jiaotong Liverpool University, China (email: nan.zhang@xjtlu.edu.cn).

Siwei Gan is with the Department of Mathematical Sciences, Xi'an Jiaotong Liverpool University, China (email: siwei.gan10@xjtlu.edu.cn). an alternative interest rate, the process of which is specified by a stochastic model. However, a drawback of the work [10] and [11] is that it does not consider the effect of transaction costs. Such drawback makes the application of the algorithm in real market is rather limited. Thus our current work is an natural expansion of the recent theoretical and numerical results on mortgage borrower's refinancing strategy under stochastic market interest environment presented in [10] and [11].

The current study considers matching the principal payment method in the financial market. In addition, we would like to emphasize the following three commonly adopted practices in mortgage industry. First, the debtor is allowed to refinance only once after the contract is signed before the expiry date. Under this assumption, the debtor should grasp the best opportunity of the lower enough interest rate to minimize the total payment. Second, if a rational debtor chooses to refinance, he is no longer responsible for the subsequent residuary interest upon refinancing and the new debt is the outstanding loan balance inherited from the old debt. This assumption is essential since otherwise the debtor will have no motivation to refinance. Third, for convenience, we assume that the debtor chooses to refinance at the beginning of a month, and pays at the end of a month. On the other hand, the market is probably not completely efficient and liquid in the sense that the debtor is often required to pay additional refinancing fee, so the impact of transaction fee is considered in this paper.

\section{OUtLINE OF THE MdOEL}

Suppose the debtor borrows $P_{0}$ with monthly interest rate $r_{0}$ during the time period ${ }_{[0 T]}$ and repays $m_{t}$ at the beginning of each month, where $t$ denotes the $t^{\text {th }}$ month. According to matching the principal repayment method, $m_{t}$ equals to a certain portion of principal plus a decreasing value of interest.

$$
m_{t}=\frac{p_{0}}{n}+\left(1-\frac{t-1}{n}\right) P_{0} r_{0}
$$

where $n$ is the total number of payment times.

The term $\underline{P}_{0}$ could be explained as a fixed portion of principal, and $\left(1-\frac{t-1}{n}\right) P_{0} r_{0}$ is an amount of decreasing interest due to the reduction of every month. At time $k$, the debtor prefers to refinance the debt with another lender when a lower interest rate $r_{k}$ is offered. On the $k^{\text {th }}$ month, he owes 
the previous bank $P_{k}$ and has paid $A_{k}$.

$$
\begin{gathered}
P_{k}=\left(1-\frac{k-1}{n}\right) P_{0} \\
A_{k}=\sum_{i=1}^{k-1} m_{i}=P_{0}(k-1)\left(r_{0}+\frac{1}{n}-\frac{k-2}{2 n} r_{0}\right)
\end{gathered}
$$

The amount of money $P(t)$ is the new principal the debtor borrows from another bank with the interest rate $r(t)$. This transaction will last from time $k$ to time $T$. The total payment over time $[0 T]$ could be described as follows:

$$
\begin{aligned}
P(T)= & A_{k}+\sum_{i=k}^{n} m_{i} \\
= & P_{0}(k-1)\left(r_{0}+\frac{1}{n}-\frac{k-2}{2 n} r_{0}\right) \\
& +P_{k}\left[1+\frac{\left(n^{*}+1\right) r_{k}}{2}\right]
\end{aligned}
$$

where $n^{*}=n-k+1$.

On the other hand, there is another method to repay loan is to matching the repayment of principal and interest. Assume the debtor borrows $P_{0}$ with interest rate $r_{0}$ over time ${ }_{[0, T]}$ and the amount of monthly payment is kept the same. In the beginning of the contract, the interest accounts for most of payment due to a large amount of loan while principal is small. Let $P(t)$ denote the amount of money owed at time $t$ and $m$ is the monthly payment.

The monthly payment $m$ should be:

$$
m=\frac{P_{0} r_{0}\left(1+r_{0}\right)^{n}}{\left(1+r_{0}\right)^{n}-1}
$$

where $n$ is the number of total repayment times.

At time $k$, the debtor owes the $P(k)$ to the previous bank. Again, due to the lower interest rate $r_{k}$, the debtor would borrow $P(k)$ from another bank to repay the remaining debts $P(k)$.

$$
P(k)=\frac{m}{r_{0}}\left[1-e^{r_{0}(k-T-1)}\right] \text { or } P(k)=\frac{m}{r_{0}}\left[1-\left(1+r_{0}\right)^{k-n-1}\right]
$$

From time $k$ to time $T$, the monthly payment should be $m_{2}$, where

$$
m_{2}=\frac{P_{k} r_{k}\left(1+r_{k}\right)^{n-k+1}}{\left(1+r_{k}\right)^{n-k+1}-1}
$$

Throughout this paper we assume that the market interest rate follows the Vasick spot interest rate process. This is to keep consistent and is a mathematical model describing the evolution of interest rate. The model specifies that the instantaneous interest rate follows the stochastic differential equation:

$$
d r_{t}=k\left(\theta-r_{t}\right) d t+\sigma d W_{t}
$$

where $k$ is the reversion rate, $\theta$ is the long-term mean interest rate and $\sigma$ is the standard deviation, all of while are positive constants. We let $r_{t}$ denote the instantaneous spot rate at time $t$, and $W_{t}$ is the standard Brownian motion. The explicit solution to $r_{t}$ from equation ( 3 ) is

$$
r_{t}=e^{-k t} r_{0}+\theta\left(1-e^{-k t}\right)+\sigma \int_{0}^{t} e^{-k(t-s)} d W_{t}
$$

Both equation (3) and equation (4) can be used equivalently to describe the alternative mortgage rate that a loan borrower may choose from the open market. But the following Euler approximation to the equation (3) is used more frequently when simulation is concerned.

$$
\Delta r=k\left(\theta-r_{t}\right) \Delta t+\sigma \Delta W_{t}
$$

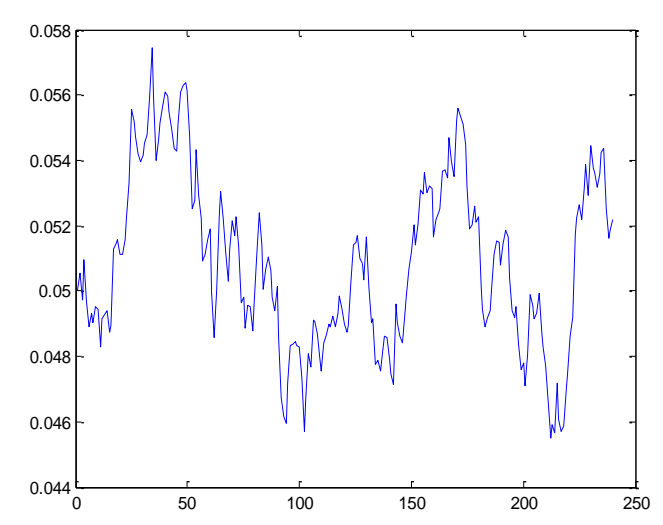

Fig. 1. Example of vasicek interest rate process

\section{THE AlgORITHM AND RESUlT}

We are looking for the optimal interest rate, for any time given, at which it is optimal to refinance. The optimal threshold is defined as the interest rate at which the likelihood to refinance in the interval $P=[90.2 \%, 90.4 \%]$. Since the likelihood is computed in statistics, it is necessary to simulate a considerable many interest rate curves so as to converge to a relatively stable value considering the randomness of the Vasicek Model. The algorithm to accomplish this, as discussed in [10] and [11], are as follows:

\section{Part 1: Initial guess.}

The Binary Search Method is used to find the optimal interest rate.

Suppose that the optimal interest rates at the $k^{\text {th }}$ year $\left(r_{k}\right)$ and the $k-1^{\text {th }}$ year $\left(r_{k-1}\right)$ have been obtained.

Let $F(x)$ denotes the probability to refinance when interest rate is $x$. The probability is got by 50,000 times simulations.

Step 1: The upper bound: $u=r_{k-1}$

The lower bound: $l=2 r_{k-1-} r_{k}\left(\right.$ Let $\left.r_{k^{-}} r_{k-I=} r_{k-I^{-}} l\right)$;

Step 2: $j=(u+l) / 2$,

If $F(j)$ is located in the interval $P$, then $r_{k-2}=j$;

Step 3: Else if $F(j)<90.2$

Then the new upper bound $u=j$

Else if $F(j)>90.4$

Then the new lower bound $l=j$.

Go back to the Step 2 until the value of $j$ converges to the 
optimal interest rate;

Step 4: Repeat the step 1-3 to obtain the optimal interest rate on specific date and get the boundary of the interest rate. To simplify the problem, we only consider the interest rate in each year.

Since $\mathrm{F}(j)$ is computed by simulation, the optimal interest rates obtained by part 1 are likely unstable and the boundary line is rough. Therefore, to modify and enhance the accuracy of each point is necessary.

Part 2: The Modified Scheme for Step 1 and 2

Step 1: Check whether the value of $F\left(r_{k}\right)$ is located in the interval $\mathrm{P}$ for continuously 3 times. If it does, the interest rate $\mathrm{r}_{\mathrm{k}}$ is considered as the eligible one, else go the next step;

Step 2: If $F\left(r_{k}\right)>90.4$

Then $r_{k=} r_{k}-0.001$

Else if $F\left(r_{k}\right)<90.2$

Then $r_{k=} r_{k}+0.001$.

Repeat the Step 1-2 to get a more accurate value of $r_{k}$.

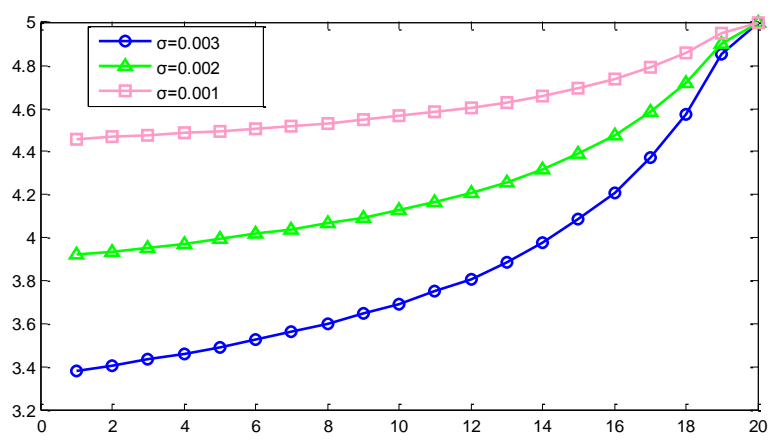

Fig. 2. The boundary of the optimal interest rate when $\theta=0.05, k=0.1$ and $r_{20}=0.05$.

In this paper, we provide a modified method for obtaining optimal refinancing interest rate boundary $r^{*}$. Compared to [10] and [11], the method in this research has two advantages. One is the short computing time, and the other one is that we consider the risk-neutral instead of setting a probability interval. Under the same assumptions as the previous research, we design the following calculation method which is based on the Monte Carlo simulation. It should be noted that the boundary is computed by flowing backwards with time. Specifically, $k=0$ expresses the expiration date, while $k=T$ refers to the time when the contract is signed.

We first consider when $k=2$, which means the remaining time for the contract is 2 months. At $k=2$, the debtor will decide whether is rational to refinance or not. We can obtain the total payment of the last two months, denoted as $T P_{2}$ in two kinds of situation - 'refinance' or 'do not refinance'. If the debtor chooses to refinance at $k=2$, the new interest rate for the last two months changes to $r_{2}$. If not, the debtor still has one chance at $k=1$, and the interest rate of the last month is based on the actual interest rate of the last month $r_{1}$ and the optimal interest rate value $r_{1}{ }^{*}$. We choose the smaller one to compute the total payment of the last two months. And the procedure can be repeated for $k=1,2,3 \ldots 240$. The formula of total payment can be expressed in general case:

$$
T P_{k}=\left\{\begin{array}{c}
\frac{k}{n} P_{0}+P_{0} r_{k} \sum_{i=1}^{k} \frac{i}{n} \\
\frac{k}{n} P_{0}+P_{0} r_{0} \sum_{i=j+1}^{k} \frac{i}{n}+P_{0} r_{j} \sum_{i=0}^{j} \frac{i}{n}
\end{array}\right.
$$

where $j$ is the time where interest rate is the first time less than or equal to the optimal refinancing interest rate boundary from the $k-1$ month to the last month. If the condition cannot be satisfied, $j$ equals to 0 , and the original contract will continue until its expiration date.

We will explain the formula of these last $k$ months' total payment when the debtor does not refinance. If the actual interest rate is firstly less than or equal to the optimal refinancing interest rate boundary, the debtor should refinance immediately. If the actual interest rate always greater than the optimal refinancing interest rate boundary, the debtor does not refinance in the overall process. Under the two kinds of situations, the optimal point can be obtained by making the two formulas of $T P_{k}$ be equal. We obtain the optimal interest rate at $k$.

Considering refinancing as a financial derivative instrument, we obtain its value, defined as $Q$, from the perspective of risk-neutral pricing as follows.

$$
\begin{aligned}
Q & =\left\{\begin{array}{cc}
P_{0}+P_{0} r_{0} \sum_{i=1}^{n} \frac{i}{n}-\left[P_{0}+P_{0} r_{0} \sum_{i=j+1}^{n} \frac{i}{n}+P_{0} r_{j} \sum_{i=1}^{j} \frac{i}{n}\right] & j \geq 1 \\
0 & j=0
\end{array}\right. \\
& =\left\{\begin{array}{cc}
P_{0}\left(r_{0}-r_{j}\right) \sum_{i=1}^{j} \frac{i}{n} & j \geq 1 \\
0 & j=0
\end{array}\right.
\end{aligned}
$$

which implies

$$
\begin{aligned}
\frac{Q}{P_{0}} & =\left\{\begin{array}{cc}
\frac{j(j+1)}{12 \times 2 \times n}\left(r_{0}-r_{j}\right) & j \geq 0 \\
0 & j=0
\end{array}\right. \\
& =\left\{\begin{array}{cc}
\frac{j(j+1)}{24 n}\left(r_{0}-r_{j}\right) & j \geq 0 \\
0 & j=0
\end{array}\right.
\end{aligned}
$$

TABle I: The Value of $\underline{Q}$ Through 10 Times of Simulation When $\overline{P_{0}}$

$$
R 0=0.05
$$

\begin{tabular}{llllll}
\hline \hline$\frac{Q}{P_{0}}$ & 0.0196 & 0.0196 & 0.0197 & 0.0197 & 0.0197 \\
\cline { 2 - 6 } & 0.0197 & 0.0197 & 0.0196 & 0.0199 & 0.0197 \\
\hline \hline
\end{tabular}

We include the transaction fee into our implementation and discussion. It is reasonable to let $\mathrm{Q}$ be a transaction fee, which means the intrinsic value of the refinancing option is zero. By choosing $\alpha=\frac{Q}{P_{0}}=0.0197$, we can obtain 


$$
T P_{k}=\left\{\begin{array}{cc}
\frac{k}{n} P_{0}+P_{0} r_{k} \sum_{i=1}^{k} \frac{i}{n}-\alpha P_{0} & \text { yes } \\
\left\{\begin{array}{cc}
\frac{k}{n} P_{0}+\sum_{i=j+1}^{k} \frac{i}{n} P_{0} r_{0}+\sum_{i=1}^{j} \frac{i}{n} P_{0} r_{j}-\alpha P_{0} & j \geq 1 \\
\frac{k}{n} P_{0}+\sum_{i=1}^{k} \frac{i}{n} P_{0} r_{0} & \text { else }
\end{array}\right. & \text { no }
\end{array}\right.
$$

Then from equation (9), we obtain

$$
\begin{aligned}
r_{k} & = \begin{cases}\frac{r_{0} \sum_{i=j+1}^{k} i+r_{j} \sum_{i=1}^{j} i}{\sum_{i=1}^{k} i} & j \geq 1 \\
\frac{\sum_{i=1}^{k} i-1}{\sum_{i=1}^{k} i} r_{0} & \text { else }\end{cases} \\
& = \begin{cases}\frac{(k+j+1)(k-j) r_{0}+(1+j) j r_{j}}{(k+1) k} & j \geq 1 \\
\frac{(k+2)(k-1)}{(k+1) k} & \text { else }\end{cases}
\end{aligned}
$$

To compare, we use the traditional method, in which the transaction fee is computed as the percentage of the outstanding balance [1], say $2 \%$.

$$
T P_{k}=\left\{\begin{array}{c}
\frac{k}{n} P_{0}+P_{0} r_{k} \sum_{i=1}^{k} \frac{i}{n}+\frac{\alpha k P_{0}}{n} \\
\frac{k}{n} P_{0}+P_{0} r_{0} \sum_{i=j+1}^{k} \frac{i}{n}+P_{0} r_{j} \sum_{i=1}^{j} \frac{i}{n}+\frac{\alpha j P_{0}}{n}
\end{array}\right.
$$

From equation (10), we obtain

$$
\begin{aligned}
r_{k} & =\left\{\begin{array}{cc}
\frac{r_{0} \sum_{i=j+1}^{k} i+r_{j} \sum_{i=1}^{j} i+\alpha(j-k)}{\sum_{i=1}^{k} i} & j \geq 1 \\
\frac{r_{0} \sum_{i=1}^{k} i-k \alpha}{\sum_{i=1}^{k} i} & \text { else }
\end{array} \quad j \geq 1\right. \\
& =\left\{\begin{array}{cl}
\frac{(k+j+1)(k-j) r_{0}+(j+1) j r_{j}+\alpha(j-k)}{(k+1) k} & \text { else }
\end{array}\right.
\end{aligned}
$$

And the boundary conditions obtained from each method showed in the following Fig. 3.

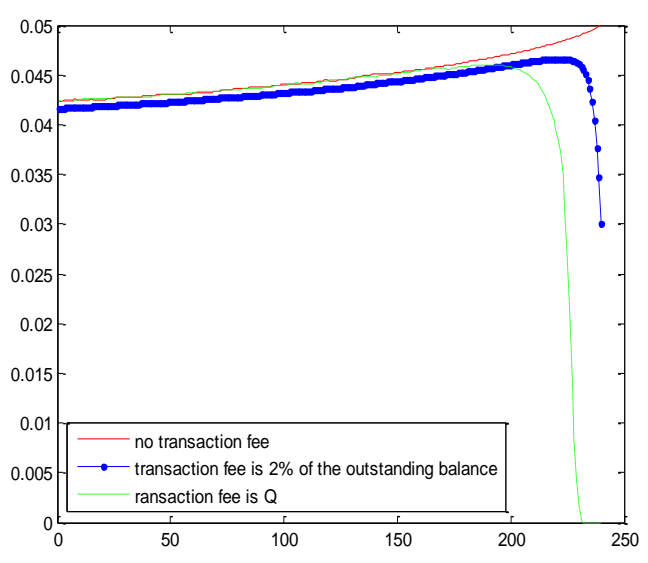

Fig. 3. The boundary conditions obtained from each method.

\section{CONCLUSION}

The current work aims to address one of the main weaknesses seen in recently developed optimization methods for finding the mortgage borrower's optimal refinancing strategy, namely, the Monte Carlo simulation based approaches contained in [10] and [11], where transaction cost is not considered. Interesting mathematical properties of the free boundary near expiry is discovered. Such property can provide useful hints for asymptotic properties of American put option as discussed in [7]. The risk neutral refinancing premium is introduced to mathematically calibrate the threshold rate at which refinancing is optimal when transaction cost is considered. Numerical results are provided to calibrate the optimal refinancing boundary.

\section{REFERENCES}

[1] A. H. Chen and D. C. Ling, "Optimal Mortgage Refinancing with Stochastic Interest Rates,” AREUEA Journal, vol. 17, no. 3, 1989.

[2] F. Agarwal, J. Driscoll, and D. Laibson, "Optimal Mortgage Refinancing: A Closed Form Solution," Federal Reserve Board or the Federal Reserve Bank of Chicago, March. 2008.

[3] D. Xie, X. Chen, and J. Chadam, "Optimal Payment of Mortgages," European Journal of Applied Mathematics, vol. 18, pp. 363-388, 2007

[4] D. Xie, "An Integral Equation Approach to Pricing Fixed Rate Mortgages," Far East Journal of Applied Mathematics, vol. 35, no. 2, pp. 233-242, 2009

[5] X. Feng and D. Xie, "Bayesian Estimation of CIR Model," Journal of Data Science, vol. 10, pp. 271-280, 2012.

[6] O. Vasicek, "An Equilibrium Characterisation of the Term Structure," Journal of Financial Economics, vol. 18, pp. 177-188, 1977.

[7] D. Xie, D. Edwards, G. Schleiniger, and Q. Zhu, "Characterization of American Put Option Using Convexity," Applied Mathematical Finance, vol. 18, pp. 353-365, 2011.

[8] F. J. Fabozzi, The Handbook of Mort-gage Backed Securities. New York: McGraw-Hill, 2001.

[9] Y. Demyanyk and O. V. Hemert. Understanding the Subprime Mortgage Crisis. Review of Financial Studies. [Online]. Available: SSRN: http://ssrn.com/abstract= 1020396.

[10] S. Gan, J. Zheng, X. Feng, and D. Xie, "When to Refinance Mortgage Loans in a Stochastic Interest Rate Environment," in Proceedings of The International Multi Conference of Engineers and Computer Scientists 2012, IMECS 2012, Hong Kong, 2012, pp. 1678-1683.

[11] J. Zheng, S.Gan, X. Feng, and D. Xie, "Optimal Mortgage Refinancing Based on Monte Carlo Simulation," IAENG International Journal of Applied Mathematics, May, 2012. 


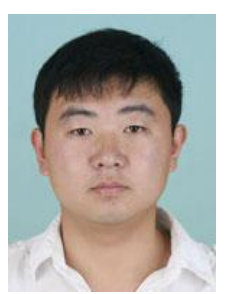

Di Jin was born in Shen Yang in 1988. He is currently a graduate student at the Department of Mathematics, the School of Mathematics and Statistics, Xi'an Jiaotong University. He holds a BS degree from the Hangzhou Dianzi University, Hangzhou, China, in 2011. His current research areas are computational mathematics and financial mathematics.

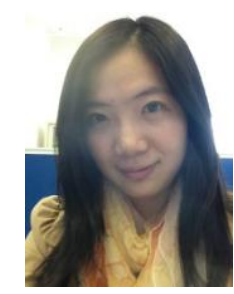

Jin Zheng was born in Yichang in 1988. She is currently a Ph.D. candidate at the Department of Mathematical Sciences, University of Liverpool. Her research areas include volatility models, option pricing, and mortgage security valuation.

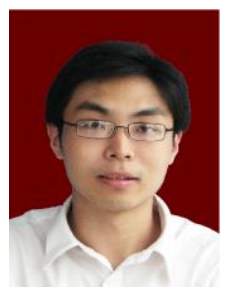

pricing.

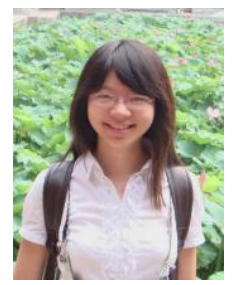

Nan Zhang was born in Tian Jin in 1979 . He is a lecturer in the Department of Computer Science and Software Engineering at Xi'an Jiaotong-Liverpool University. He has a Ph.D. and a M.Sc. from the School of Computer Science, the University of Birmingham UK, and a B.Eng. degree in computer science from the University of Shandong China. His research interests focus on high-performance parallel computing and its applications on financial derivative

Siwei Gan was born in Guangxi in 1991. She is currently an undergraduate student of financial mathematics at the Department of Mathematics, the School of Science, Xi'an Jiaotong Liverpool University. 'When to Refinance Mortgage Loans in a Stochastic Interest Rate Environment' Proceedings of The International MultiConference of Engineers and Computer Scientists, 2012. 'Optimal Mortgage Refinancing Based on Monte Carlo Simulation'

IAENG International Journal of Applied MathematFics, 2012.

Her current research interests involve Monte Carlo simulation, Mortgage loans and American options. 